\title{
MINIMAL UNIT VECTOR FIELDS
}

\author{
Olga GIL-MEDRANO ${ }^{1}$ AND Elisa LlinARES-Fuster ${ }^{2}$
}

(Received March 27, 2000, revised April 4, 2001)

\begin{abstract}
We compute the first variation of the functional that assigns each unit vector field the volume of its image in the unit tangent bundle. It is shown that critical points are exactly those vector fields that determine a minimal immersion. We also find a necessary and sufficient condition that a vector field, defined in an open manifold, must fulfill to be minimal, and obtain a simpler equivalent condition when the vector field is Killing. The condition is fulfilled, in particular, by the characteristic vector field of a Sasakian manifold and by Hopf vector fields on spheres.
\end{abstract}

1. Introduction. Let $M$ be a Riemannian manifold such that the set $\mathcal{X}^{1}(M)$ of unit vector fields is not empty. In [GlZi], where $M$ is also assumed to be compact, oriented and boundaryless, the volume of an element $V \in \mathcal{X}^{1}(M)$ was defined to be the volume of the submanifold $V(M)$, which is the image of the immersion $V: M \rightarrow T^{1} M$, when the unit tangent bundle $T^{1} M$ is equipped with the restriction of the Sasaki metric. There is a trivial absolute minimum of the volume functional when unit parallel vector fields exist, but this is not always the case, since such a vector field will determine two mutually orthogonal complementary totally geodesic foliations.

On a round unit odd-dimensional sphere, Gluck and Ziller ([GlZi]) considered Hopf vector fields as the candidates for this absolute minimum and showed that it is the case for the three-sphere $S^{3}$. Their method of calibrated geometries cannot be applied to higher dimensional spheres and in fact, Johnson ([Jo]) showed that the Hopf vector fields on $S^{5}$ are not local minima of the volume. He used direct methods to show that for every deformation of a Hopf vector field the first variation vanishes but that there are deformations on which the second variation is negative. In both papers the results are derived using the specific properties of Hopf vector fields and spheres.

For a given $M$, unit vector fields of minimum volume, if they exist, are to be found among the critical points of the volume functional restricted to $\mathcal{X}^{1}(M)$. In order to characterize these critical points we have computed the first variation of the functional and have associated to each $V \in \mathcal{X}^{1}(M)$ a 1 -form which vanishes when and only when $V$ is critical. This 1 -form depends on the second covariant derivative of $V$.

2000 Mathematics Subject Classification. Primary 53C20; Secondary 53C25, 53C42.

Key words and phrases. Volume of vector fields, critical points, minimal vector fields, Killing vector fields, Hopf vector fields, Sasakian manifolds.

${ }^{1}$ Partially supported by DGES Grant No. PB97-1425.

${ }^{2}$ Supported by Ministerio de Educación y Cultura Research Grant. 
It is clear that if a vector field defines a minimal immersion, it should be a solution of the variational problem restricted to $\mathcal{X}^{1}(M)$, but we have shown that the converse is also true:

An element $V \in \mathcal{X}^{1}(M)$ is a critical point of the volume functional restricted to $\mathcal{X}^{1}(M)$ if and only if $V: M \rightarrow\left(T^{1} M, g^{S}\right)$ is a minimal immersion.

In [Pe], Pedersen constructed, for any dimension, unit vector fields of exceptionally small volume, on the open manifold $U$ defined to be the sphere minus one point. In particular, for $S^{2 m+1}$ with $m \geq 2$, their volume is lower than the volume of Hopf vector fields, and she conjectured that this value is the infimum (not attained) of the volume of unit vector fields on $S^{2 m+1}$. In this paper, we use a direct computation of the involved tensor field to show that these vector fields define minimal immersions of $U$ into its unit tangent bundle.

Hopf vector fields on spheres define a totally geodesic Riemannian foliation with bundlelike metric or, equivalently, they are unit Killing vector fields; many results of this paper concern this class of vector fields. For instance, we prove the following:

Let $M$ be a manifold of constant sectional curvature $k$. Every unit Killing vector field is minimal and its volume is equal to $(k+1)^{(n-1) / 2} \operatorname{vol}(M)$, where $n=\operatorname{dim} M$.

It is worth noting that under the hypotheses, the curvature must be nonnegative; if the manifold is flat, only the trivial case of a parallel vector field is admissible and if $k>0$, the dimension of the manifold should be odd. Therefore, apart from flat spaces, the only complete manifolds involved are the quotients of round spheres. Since it is known that Hopf vector fields on $S^{2 m+1}$ have volume $2^{m} \operatorname{vol}\left(S^{2 m+1}\right)$ ([GlZi]) and that they are critical ([Jo]), for complete manifolds, our result could be seen as a slight generalization and an extremely simple new proof of these results.

The merit is, however, that as we only use the properties of the curvature tensor, on a manifold with such a vector field, the method is purely local and no completeness assumption is needed.

In a general manifold, the existence of a unit Killing vector field does not imply any restriction on the dimension and only sectional curvatures of planes containing $V$ have to be nonnegative. We have found that the necessary and sufficient condition for a unit Killing vector field $V$ to be minimal is the vanishing of certain 1-form given in terms of the covariant derivative of $V$ and the curvature tensor.

It is commonly said concerning this problem that best organized vector fields are rewarded with small volume; we can see now that even for a well-organized vector field it is necessary, at least, to be well-adapted to the ambient.

The curvature condition is satisfied by parallel vector fields and for every unit Killing vector field in a constant curvature space. Using our characterization we show that the characteristic vector field of a Sasakian manifold is always minimal.

In general, every unit Killing vector field $V$ such that the curvature tensor $R$ verifies $R(X, Y, V)=0$ for all vector fields $X, Y$ orthogonal to $V$ is minimal. The necessary and sufficient condition is more general, as we show with an example of an invariant vector field in the generalized Heisenberg group. 
We have devoted the last part of the paper to show how the condition can be written for three-dimensional and four-dimensional manifolds; in both cases it is equivalent to $\rho_{V}(X)=$ $\{0\}$ for all vector fields $X$ orthogonal to $V$, where $\rho_{V}$ is the 1 -form related to the Ricci tensor $\rho$ by $\rho_{V}(X)=\rho(X, V)$. The proof makes use of the hypothesis on the dimension in a very specific form, so the result cannot be generalized to higher dimensions.

These characterizations have been used by several authors in [BoVa1], [BoVa2], [GMGDVa], [GDVa1], [GDVa2], [GDVa3], [TsVa1], [TsVa2] [TsVa3], to obtain many examples of minimal unit vector fields.

The authors want to express their gratitude to Fabiano Brito, Antonio Ros and Lieven Vanhecke for helpful comments.

2. First variation of the volume functional in the space of unit vector fields. If $(M, g)$ is a smooth, connected, closed Riemannian manifold, then the set $\mathcal{X}^{1}(M)$ of all smooth unit vector fields, if nonempty, can be endowed with a structure of Fréchet manifold, compatible with its $C^{\infty}$-topology, such that each $V \in \mathcal{X}^{1}(M)$ is contained in a chart modeled in $\mathcal{H}^{V}$, the space of smooth vector fields in the horizontal distribution determined by $V$ and the metric. In fact, $\mathcal{X}^{1}(M)$ is a Fréchet submanifold of the Fréchet space $\mathcal{X}(M)$ (see [GM]).

The volume $F(V)$ of an element $V \in \mathcal{X}^{1}(M)$ is defined to be the volume of the submanifold $V(M)$, which is the image of the immersion $V: M \rightarrow T^{1} M$, when in $T^{1} M$ the usual metric $g^{S}$, defined by Sasaki, is considered. It can be described as the volume of $M$ with the induced metric $h_{V}=V^{*} g^{S}$, which is related with $g$ and the $(1,1)$-tensor field $\nabla V$ as follows:

$$
\begin{aligned}
h_{V}(X, Y) & =\left(V^{*} g^{S}\right)(X, Y)=g^{S}(T V \circ X, T V \circ Y) \\
& =g(T \pi \circ T V \circ X, T \pi \circ T V \circ Y)+g(\kappa \circ T V \circ X, \kappa \circ T V \circ Y) \\
& =g(X, Y)+g\left(\nabla_{X} V, \nabla_{Y} V\right)=g(X, Y)+g\left(\left((\nabla V)^{t} \circ(\nabla V)\right)(X), Y\right),
\end{aligned}
$$

where $\pi: T^{1} M \rightarrow M$ is the projection and $\kappa$ denotes the connection map of the Levi-Civita connection of $g$. If we consider the $g$-symmetric $(1,1)$-tensor field $L_{V}=\operatorname{Id}+(\nabla V)^{t} \circ \nabla V$, then $h_{V}(X, Y)=g\left(L_{V}(X), Y\right)$.

A map $f: \mathcal{X}^{1}(M) \rightarrow C^{\infty}(M)$ can be defined as $f(V)=\sqrt{\operatorname{det} L_{V}}$, by which the volume functional $F: \mathcal{X}^{1}(M) \rightarrow \boldsymbol{R}$ is given by

$$
F(V)=\int_{M} f(V) d v,
$$

where $d v$ is the density on $M$ defined by $g$.

Let us compute the first variation of $F$. We will denote the differential, or the tangent map, of $F$ at $V$ as $T_{V} F$.

Proposition 1. Let $V \in \mathcal{X}^{1}(M)$ be a unit vector field and $A \in T_{V} \mathcal{X}^{1}(M)=\mathcal{H}^{V}$ be a tangent vector. The tangent map of $F$ at $V$ acting on $A$ is given by

$$
\left(T_{V} F\right)(A)=\int_{M}\left(T_{V} f\right)(A) d v=\int_{M} f(V) \operatorname{tr}\left(L_{V}^{-1} \circ(\nabla V)^{t} \circ \nabla A\right) d v .
$$


Proof. Let $V: I \rightarrow \mathcal{X}^{1}(M)$ be a curve for some open interval $I$ containing 0 , such that $V(0)=V$ and $V^{\prime}(0)=A$. Take $L(t)=L_{V(t)}$. Then $L^{\prime}(0)=(\nabla A)^{t} \circ \nabla V+(\nabla V)^{t} \circ \nabla A$ and therefore

$$
\left(T_{V} f\right)(A)=(f \circ V)^{\prime}(0)=\frac{1}{2} f(V) \operatorname{tr}\left(L^{\prime}(0) \circ L^{-1}(0)\right)=f(V) \operatorname{tr}\left(L_{V}^{-1} \circ(\nabla V)^{t} \circ \nabla A\right) .
$$

To write the tangent map, and therefore the condition for a vector field to be critical, in a simpler form, we will use the following Lemma, the proof of which is a straightforward application of the definitions.

LEMMA 2. Given a $(1,1)$-tensor field $K$, let $\omega_{K}$ be the 1-form defined by $\omega_{K}(X)=$ $\operatorname{tr}\left(Z \mapsto\left(\nabla_{Z} K\right)(X)\right)$. For each $A \in \mathcal{X}(M)$ we have

$$
\omega_{K}(A)=-\operatorname{tr}(K \circ \nabla A)-\delta \alpha,
$$

where $\delta$ represents the divergence operator of $g$ and $\alpha(X)=g(K(A), X)$.

COROllary 3. Let $V \in \mathcal{X}^{1}(M)$ be a unit vector field and $A \in T_{V} \mathcal{X}^{1}(M)=\mathcal{H}^{V}$ be a tangent vector. The tangent map of $F$ at $V$ acting on $A$ can be written as

$$
\left(T_{V} F\right)(A)=-\int_{M} \omega_{V}(A) d v
$$

where $\omega_{V}(X)=\operatorname{tr}\left(Z \mapsto\left(\nabla_{Z} K_{V}\right)(X)\right)$ and $K_{V}=f(V) L_{V}^{-1} \circ(\nabla V)^{t}$.

PROPOSITION 4. A unit vector field $V \in \mathcal{X}^{1}(M)$ is a critical point of $F$ if and only if the 1-form $\omega_{V}$ annihilates $\mathcal{H}^{V}$, or equivalently, if and only if the vector field $X_{V}$, given by $\omega_{V}(X)=g\left(X_{V}, X\right)$, is in the distribution $\mathcal{V}$ determined by $V$.

PROOF. If $V$ is a critical point and if we represent by $X_{V}^{h}$ the projection of $X_{V}$ onto $\mathcal{H}^{V}$, then

$$
0=\left(T_{V} F\right)\left(X_{V}^{h}\right)=-\int_{M} \omega_{V}\left(X_{V}^{h}\right) d v .
$$

Now, $\omega_{V}\left(X_{V}^{h}\right)=g\left(X_{V}^{h}, X_{V}\right)=g\left(X_{V}^{h}, X_{V}^{h}\right) \geq 0$, and then for $V$ to be critical, $X_{V}-g\left(X_{V}, V\right) V$ must vanish. The other implication is immediate.

The condition above means that $\left(T_{V} f\right)(A)=-\delta \alpha_{V A}$, where $\alpha_{V A}(X)=g\left(K_{V}(A), X\right)$ and so, if $M$ is compact with nonempty boundary, then $\omega_{V}\left(\mathcal{H}^{V}\right)=\{0\}$ if and only if for each $A \in \mathcal{H}^{V}$

$$
\left(T_{V} F\right)(A)=\int_{\partial M} f(V) g\left(A,\left(\nabla V \circ L_{V}^{-1}\right)(\eta)\right) d \bar{v} .
$$

If $M$ is noncompact, we denote by $\mathcal{T}^{*}$ the family of open sets in $M$ with compact closure; for each $U \in \mathcal{T}^{*}$, we also represent by $\mathcal{H}_{U}^{V}$ the subset of $\mathcal{H}^{V}$ consisting of those elements with support in $U$. We then have the following

LEMMA 5. $\omega_{V}\left(\mathcal{H}^{V}\right)=\{0\}$ if and only if $\left(T_{V} F^{U}\right)\left(\mathcal{H}_{U}^{V}\right)=\{0\}$ for all $U \in \mathcal{T}^{*}$, where $F^{U}: \mathcal{X}^{1}(M) \rightarrow \boldsymbol{R}$ maps each $X$ to the volume of $X(U)$. 
It is well-known that an immersion $\varphi: M \rightarrow\left(T^{1} M, g^{S}\right)$ is minimal if and only if its mean curvature vector field $\eta^{\varphi}$ vanishes and that this condition is equivalent to $\left(T_{\varphi} \tilde{F}^{U}\right)(\xi)=$ 0 for all $U \in \mathcal{T}^{*}$ and for all vector field $\xi$ on $T^{1} M$ along $\varphi$ with support in $U$. Here $\tilde{F}^{U}$ : $\operatorname{Imm}\left(M, T^{1} M\right) \rightarrow \boldsymbol{R}$ maps $\varphi$ to the volume of $\varphi(U)$.

Proposition 6. For a unit vector field $V$ on a general manifold $M$, the map $V$ : $M \rightarrow\left(T^{1} M, g^{S}\right)$ is a minimal immersion if and only if $\omega_{V}\left(\mathcal{H}^{V}\right)=\{0\}$.

PROOF. It is clear, using Lemma 5, that every $V \in \mathcal{X}^{1}(M)$ which gives rise to a minimal immersion must satisfy $\omega_{V}\left(\mathcal{H}^{V}\right)=\{0\}$. Conversely, for such a $V$, the mean curvature $\eta^{V}$ is a vector field on $T^{1} M$, along $V$, having the property that

$$
\int_{U} g^{S}\left(\eta^{V}, \xi\right) d v_{S}=\left(T_{V} \tilde{F}^{U}\right)(\xi)=0
$$

for all $U \in \mathcal{T}^{*}$ and for all vector fields $\xi$ along $V$, with support in $U$, that are tangent to variations in the submanifold $\mathcal{X}^{1}(M)$ of the manifold of all immersions of $M$ into $T^{1} M$. Here $d v_{S}$ is the density in $M$ defined by $V^{*} g^{S}$.

It is not difficult to show that such a vector field is just a section, along $V$, of the vertical bundle of the bundle $\pi: T^{1} M \rightarrow M$; that is, for each $p \in M, \xi_{p} \in T_{V(p)}\left(T_{p}^{1} M\right)=$ $\operatorname{ker}\left(T_{V(p)} \pi\right)=\operatorname{Vert}_{V(p)}$. Therefore, $\omega_{V}\left(\mathcal{H}^{V}\right)=\{0\}$ if and only if the mean curvature vector field verifies $\eta_{p}^{V} \in \operatorname{Vert}_{V(p)}^{\perp}$ for all $p \in M$.

Since for every unit vector field $T_{V(p)}\left(T^{1} M\right)=\left(\operatorname{Im} T_{p} V\right) \oplus \operatorname{ker}\left(T_{V(p)} \pi\right)$ and for every immersion $\eta_{p}^{V} \in\left(\operatorname{Im} T_{p} V\right)^{\perp}$, if $\omega_{V}\left(\mathcal{H}^{V}\right)=\{0\}$, then $\eta_{p}^{V}$ must vanish.

It will be useful to write the condition for a unit vector field to be minimal in terms of local adapted orthonormal frames. Here for a given $V \in \mathcal{X}^{1}(M)$, a locally defined orthonormal frame field $\left\{E_{i}\right\}_{i=1}^{n}$ will be said to be adapted if $E_{n}=V$.

If we denote $G_{i j}^{k}=g\left(\nabla_{E_{i}} E_{j}, E_{k}\right)$, then $(\nabla V)_{i}^{j}=G_{i n}^{j}$, and using the fact that $G_{i j}^{k}=$ $-G_{i k}^{j}$, we obtain $(\nabla V)_{i}^{n}=0$. Since the 1 -form $\omega_{V}$ can be computed as

$$
\omega_{V}(X)=\sum_{j} g\left(\left(\nabla_{E_{j}} K_{V}\right)(X), E_{j}\right),
$$

it is easy to see that $\omega_{i}=\omega_{V}\left(E_{i}\right)=\sum_{j}\left\{E_{j}\left(K_{i}^{j}\right)+\sum_{k}\left(G_{j k}^{j} K_{i}^{k}-G_{j i}^{k} K_{k}^{j}\right)\right\}$, where $K_{i}^{j}=$ $g\left(K_{V}\left(E_{i}\right), E_{j}\right)$. Hence we have proved

PROPOSITION 7. A unit vector field is minimal if and only if

$$
\sum_{j}\left\{E_{j}\left(K_{i}^{j}\right)+\sum_{k}\left(G_{j k}^{j} K_{i}^{k}-G_{j i}^{k} K_{k}^{j}\right)\right\}=0,
$$

for all $i \in\{1, \ldots, n-1\}$.

3. Minimal unit vector fields in constant curvature spaces. The first example of these vector fields is given by a vector field defined only on a noncomplete manifold consisting of the sphere $S^{n}$ minus one point. 
In [Pe], for $v_{0} \in T_{p_{0}} S^{n}$, a vector field $V$ is defined by taking $V(p)$ as the element of $T_{p} S^{n}$ obtained by parallel translating $v_{0}$ along the great circle of $S^{n}$ passing through $p_{0}$ and $p$. Then, $V$ is defined only on $M=S^{n}-\left\{-p_{0}\right\}$. To compute the tensor field involved in the condition for a vector field to be minimal, it will be useful to have the explicit expression of $V$. To this end, we may assume, without lost of generality, that $p_{0}=N=(0, \ldots, 1)$ and so $M=S^{n}-\{S\}$ with $S=(0, \ldots,-1)$. Then it is easy to see the following

Lemma 8. Let $v_{0} \in T_{N} S^{n}$ be a unit vector. Then the corresponding vector field $V$ in $M$ is given by

$$
V(p)=\left\langle v_{o}, \bar{p}\right\rangle\left(h(p) \bar{p}-\left(\frac{\partial}{\partial r^{n+1}}\right)_{p}\right)+v_{0},
$$

where $\left\{r^{i}\right\}_{i=1}^{n+1}$ represent the usual coordinates on $\boldsymbol{R}^{n+1}, h(p)=-\left(1+p_{n+1}\right)^{-1}$ and $\bar{p}=$ $\sum_{k=1}^{n} p_{k}\left(\partial / \partial r^{k}\right) p_{\text {. }}$

PROPOSITION 9. Any vector field defined as above is minimal.

PROOF. Let $\left\{e_{i}\right\}_{i=1}^{n+1}$ be the canonical basis of $\boldsymbol{R}^{n+1}$. We assume, for simplicity, that $v_{0}=e_{n}$. The vector fields $\left\{E_{i}\right\}_{i=1}^{n}$, obtained by the procedure described above, give rise to a local adapted orthonormal frame. According to Proposition 7, we need to show that $\omega_{i}=0$ for $i \in\{1, \ldots, n-1\}$, where

$$
\omega_{i}=\sum_{j=1}^{n}\left\{E_{j}\left(K_{i}^{j}\right)+\sum_{k=1}^{n}\left(G_{j k}^{j} K_{i}^{k}-G_{j i}^{k} K_{k}^{j}\right)\right\} .
$$

By elementary computations using that

$$
E_{j}=\sum_{k=1}^{n}\left(\delta_{j k}+h r^{j} r^{k}\right) \frac{\partial}{\partial r^{k}}-r^{j} \frac{\partial}{\partial r^{n+1}}
$$

and that $h^{2} \sum_{k=1}^{n}\left(r^{k}\right)^{2}+1=-2 h$ on $M$, we have

$$
G_{i j}^{k}=h\left(\delta_{i k} r^{j}-\delta_{i j} r^{k}\right) \quad \text { with } \quad i, j, k \in\{1, \ldots, n\} .
$$

As a consequence, we obtain

$$
\omega_{i}=\sum_{j=1}^{n} E_{j}\left(K_{i}^{j}\right)+h\left\{(n-1) \sum_{k=1}^{n} K_{i}^{k} r^{k}-r^{i} \sum_{k=1}^{n} K_{k}^{k}+\sum_{k=1}^{n} K_{k}^{i} r^{k}\right\} .
$$

Let us compute the expression of $K$ with respect to this frame. The matrix of $L$ is given by

$$
\left(\begin{array}{cc}
g \text { Id } & h^{2} r^{n} a^{t} \\
h^{2} r^{n} a & h^{2}\|a\|^{2}+1
\end{array}\right)
$$

where $g=\left(h r^{n}\right)^{2}+1$ and $a=-\left(r^{1}, \ldots, r^{n-1}\right)$.

It is easy to see that for a matrix $A$ of the form

$$
\left(\begin{array}{cc}
\lambda \operatorname{Id} & b^{t} \\
b & \varepsilon
\end{array}\right)
$$


with $\lambda, \varepsilon \in \boldsymbol{R}$ and $b \in \boldsymbol{R}^{n-1}$, we have $\operatorname{det} A=\lambda^{n-2}\left(\lambda \varepsilon-\|b\|^{2}\right)$ and

$$
A^{-1}=\frac{1}{\lambda \varepsilon-\|b\|^{2}}\left(\begin{array}{cc}
\left(\left(\lambda \varepsilon-\|b\|^{2}\right) \mathrm{Id}+b^{t} b\right) / \lambda & -b^{t} \\
-b & \lambda
\end{array}\right) .
$$

Then $f=g^{(n-2) / 2}(-2 h)^{1 / 2}$ and, since $K=f L^{-1} \circ(\nabla V)^{t}$, we obtain by a straightforward computation that, for $i, j \in\{1, \ldots, n-1\}$,

$$
K_{i}^{j}=\frac{f h r^{n}}{g}\left(\delta_{i j}+\frac{h r^{i} r^{j}}{2}\right) \text { and } K_{i}^{n}=\frac{f r^{i}}{2} .
$$

Moreover, on every point of $M$ we have $\sum_{k=1}^{n} K_{i}^{k} r^{k}=0$,

$$
\sum_{k=1}^{n} K_{k}^{k}=\frac{f h r^{n}}{g}\left((n-1)+\frac{h\|a\|^{2}}{2}\right) \text { and } \sum_{k=1}^{n} K_{k}^{i} r^{k}=\frac{f h r^{n} r^{i}}{g}\left(1+\frac{h\|a\|^{2}}{2}\right) .
$$

Now, using (1), we get

$$
\omega_{i}=\sum_{j=1}^{n} E_{j}\left(K_{i}^{j}\right)+(2-n) \frac{f h^{2} r^{n} r^{i}}{g} .
$$

To conclude, we only need to compute $\sum_{j=1}^{n} E_{j}\left(K_{i}^{j}\right)$. Let us define the vector field

$$
E=\sum_{k=1}^{n} r^{k} \frac{\partial}{\partial r^{k}}-\frac{1-\left(r^{n+1}\right)^{2}}{r^{n+1}} \frac{\partial}{\partial r^{n+1}}
$$

Since this is tangent to the sphere, $E\left(\sum_{k=1}^{n} K_{i}^{k} r^{k}\right)=0$ and also $\sum_{k=1}^{n} r^{k} E\left(K_{i}^{k}\right)=0$. Furthermore,

and then

$$
E_{j}=\frac{\partial}{\partial r^{j}}-\frac{r^{j}}{r^{n+1}} \frac{\partial}{\partial r^{n+1}}-\frac{r^{j}}{1+r^{n+1}} E
$$

$$
\sum_{j=1}^{n} E_{j}\left(K_{i}^{j}\right)=\sum_{j=1}^{n} \frac{\partial}{\partial r^{j}}\left(K_{i}^{j}\right)-\frac{1}{r^{n+1}} \sum_{j=1}^{n} r^{j} \frac{\partial}{\partial r^{n+1}}\left(K_{i}^{j}\right) .
$$

By differentiation using that $h^{2}\|a\|^{2}=-(2 h+g)$, it is easy to see that

$$
\sum_{j=1}^{n} r^{j} \frac{\partial}{\partial r^{n+1}}\left(K_{i}^{j}\right)=r^{i} r^{n}\left(-\frac{f g}{2 h} \frac{\partial(h / g)}{\partial r^{n+1}}-\frac{f(2 h+g)}{2 h g} \frac{\partial h}{\partial r^{n+1}}\right)=\frac{f h^{2} r^{i} r^{n}}{g} r^{n+1} \text {. }
$$

Since

$$
\sum_{j=1}^{n} \frac{\partial}{\partial r^{j}}\left(K_{i}^{j}\right)=(n-1) \frac{f h^{2} r^{i} r^{n}}{g}
$$

we obtain $\omega_{i}=0$.

REMARK. In [Pe], it is shown that the generalized Pontryagin cycle is minimal at each smooth point as a submanifold of the corresponding Stiefel manifold. From this fact, the minimality of $V$ can be also obtained by a different method. 
In general, the minimality condition is difficult to handle. However, it becomes simpler if we assume that the vector field verifies some additional property.

LEMMA 10. A unit vector field with totally geodesic flow is minimal if and only if

$$
\sum_{j=1}^{n-1}\left\{E_{j}\left(K_{i}^{j}\right)+\sum_{k=1}^{n-1}\left(G_{j k}^{j} K_{i}^{k}-G_{j i}^{k} K_{k}^{j}\right)\right\}=0
$$

for all $i \in\{1, \ldots, n-1\}$.

Proof. If the flow of $V$ is totally geodesic, then $(\nabla V)^{t}\left(\mathcal{H}^{V}\right) \subset \mathcal{H}^{V}$ and consequently $K_{V}\left(\mathcal{H}^{V}\right) \subset \mathcal{H}^{V}$. Hence the result follows from Proposition 7 .

Let $R$ be the $(1,3)$-curvature tensor defined by

$$
R(X, Y, Z)=-\nabla_{X} \nabla_{Y} Z+\nabla_{Y} \nabla_{X} Z+\nabla_{[X, Y]} Z .
$$

We will use the same symbol for the $(0,4)$-tensor field $R(X, Y, Z, W)=g(R(X, Y, Z), W)$.

LEMMA 11. If $V$ is a unit Killing vector field, then $(\nabla V)^{t} \circ \nabla V=R(V, \cdot, V)$.

PRoOF. It is easy to see that, for a unit Killing vector field, the $(0,2)$-tensor field $h$ defined as $h(X, Y)=g(R(V, X, V), Y)$ is given by

$$
h(X, Y)=g\left(\nabla_{X} V, \nabla_{V} Y\right)-V\left(g\left(\nabla_{X} V, Y\right)\right)-g\left(\nabla_{Y} V, \nabla_{V} X\right)+g\left(\nabla_{Y} V, \nabla_{X} V\right) .
$$

Since $h$ is symmetric, $h(X, Y)$ must be equal to $g\left(\nabla_{X} V, \nabla_{Y} V\right)$ and therefore, for all $X$ in $\mathcal{X}(M), R(V, X, V)=(\nabla V)^{t} \circ(\nabla V)(X)$ as claimed.

LEMMA 12. With respect to any local orthonormal frame, the components of the curvature tensor are given by

$$
R_{j i k r}=E_{i}\left(G_{j k}^{r}\right)-E_{j}\left(G_{i k}^{r}\right)+\sum_{l=1}^{n}\left\{G_{j k}^{l} G_{i l}^{r}-G_{i k}^{l} G_{j l}^{r}-G_{i j}^{l} G_{l k}^{r}+G_{j i}^{l} G_{l k}^{r}\right\} .
$$

In particular, if $V$ is a unit Killing vector field and the frame is adapted, then, for $i, j, k \in$ $\{1, \ldots, n-1\}$

$$
\begin{aligned}
R_{j i k n}= & -E_{i}\left((\nabla V)_{j}^{k}\right)+E_{j}\left((\nabla V)_{i}^{k}\right) \\
& +\sum_{l=1}^{n-1}\left\{-G_{i l}^{k}(\nabla V)_{j}^{l}+G_{j l}^{k}(\nabla V)_{i}^{l}+G_{i j}^{l}(\nabla V)_{l}^{k}-G_{j i}^{l}(\nabla V)_{l}^{k}\right\}
\end{aligned}
$$

Proposition 13. Let $M$ be an $n$-dimensional manifold of constant sectional curvature $k$. Then every unit Killing vector field on $M$ is minimal. Moreover, $f(V)=(k+1)^{(n-1) / 2}$ and $F(V)=(k+1)^{(n-1) / 2} \operatorname{vol}(M)$.

PROOF. It follows from Lemma 11 that for every local adapted orthonormal frame, $\left((\nabla V)^{t} \circ \nabla V\right)_{i}^{j}=k\left(\delta_{i}^{j}-\delta_{n j} \delta_{n i}\right)$ and then $f=(k+1)^{(n-1) / 2}$. Moreover,

$$
K=(k+1)^{(n-3) / 2}(\nabla V)^{t}
$$


Under the hypothesis, if we put $k=j$ in the equality in Lemma 12, we obtain

$$
\begin{aligned}
E_{j}\left((\nabla V)_{i}^{j}\right) & =\sum_{l=1}^{n-1}\left\{G_{i l}^{j}(\nabla V)_{j}^{l}-G_{j l}^{j}(\nabla V)_{i}^{l}-G_{i j}^{l}(\nabla V)_{l}^{j}+G_{j i}^{l}(\nabla V)_{l}^{j}\right\} \\
& =\sum_{l=1}^{n-1}\left\{G_{j i}^{l}(\nabla V)_{l}^{j}-G_{j l}^{j}(\nabla V)_{i}^{l}\right\}
\end{aligned}
$$

and then Lemma 10 gives us the result.

REMARK. The condition for $V$ to be a unit Killing vector field is equivalent to that of $\mathcal{V}$ being a totally geodesic, Riemannian foliation with bundle-like metric. If a Riemannian manifold of constant curvature $k$ admits such a foliation $\mathcal{V}$, then $k \geq 0$, and $k=0$ would imply that $\mathcal{H}^{V}$ is also involutive; therefore the manifold would be, locally, a Riemannian product ([Ca]). On the other hand, the existence of a unit Killing vector field in a manifold of positive curvature implies that the dimension must be odd. Consequently, apart from the trivial case of parallel vector fields, the hypotheses of Proposition 13 imply $k>0$ and $n=2 m+1$. If we assume, moreover, $M$ to be complete, then $M$ should be a quotient of $S^{2 m+1}$ and, according to [GrGr], the lift of the vector field must be a Hopf vector field. In view of the corresponding results in [GlZi] and [Jo], Proposition 13 essentially provides new information only in the case where the manifold is not complete.

4. Minimal unit Killing vector fields. We have seen in Proposition 13 that every unit Killing vector field is minimal if the manifold has constant curvature. In this section we will show a necessary and sufficient condition for a unit Killing vector field to be minimal in a general manifold; the condition involves $\nabla V$ and the curvature of $M$.

For a Killing vector field $V$, the rank of $\nabla V$ must be even, and for each point of an open dense subset we can find a local adapted orthonormal frame

$$
\left\{E_{i}, E_{i^{*}}, E_{2 m+1}, \ldots, E_{n}=V\right\}_{i=1}^{m},
$$

with $2 m$ the rank of $\nabla V$, such that $\nabla V\left(E_{i}\right)=-\lambda_{i} E_{i^{*}}, \nabla V\left(E_{i^{*}}\right)=\lambda_{i} E_{i}$ for $i \in\{1, \ldots, m\}$ and $\nabla V\left(E_{\alpha}\right)=0$ for $\alpha \in\{2 m+1, \ldots, n\}$. From Lemma $11, \lambda_{i}^{2}$ is the common value of the sectional curvatures of the planes generated by $V$ and $E_{i}$ and by $V$ and $E_{i^{*}}$.

THEOREM 14. Let $V$ be a unit Killing vector field, then $\omega_{V}=f \tilde{\rho}_{V}$, where $\tilde{\rho}_{V}(X)$ is defined to be

$$
\sum_{j=1}^{n}\left(R\left(\left(L_{V}^{-1} \circ \nabla V\right)(X),\left(L_{V}^{-1} \circ \nabla V\right)\left(E_{j}\right), V, E_{j}\right)+R\left(L_{V}^{-1}(X), L_{V}^{-1}\left(E_{j}\right), V, E_{j}\right)\right) .
$$

Consequently, $V$ is minimal if and only if the 1-form $\tilde{\rho}_{V}$ annihilates $\mathcal{H}^{V}$.

Proof. It is clear that $L_{V}\left(E_{i}\right)=\left(1+\lambda_{i}^{2}\right) E_{i}, L_{V}\left(E_{i^{*}}\right)=\left(1+\lambda_{i}^{2}\right) E_{i^{*}}$ for $i \in$ $\{1, \ldots, m\}$, and $L_{V}\left(E_{\alpha}\right)=E_{\alpha}$ for $\alpha \in\{2 m+1, \ldots, n\}$. 
Since $K_{V}=f L_{V}^{-1} \circ(\nabla V)^{t}$, it follows that $K_{V}\left(E_{i}\right)=f\left(\lambda_{i} /\left(1+\lambda_{i}^{2}\right)\right) E_{i^{*}}, K_{V}\left(E_{i^{*}}\right)=$ $-f\left(\lambda_{i} /\left(1+\lambda_{i}^{2}\right)\right) E_{i}$ and $K_{V}\left(E_{\alpha}\right)=0$, for $i \in\{1, \ldots, m\}$ and $\alpha \in\{2 m+1, \ldots, n\}$. From Lemma 10, we get

$$
\begin{aligned}
& \omega_{V}\left(E_{i}\right)=E_{i^{*}}\left(K_{i}^{i^{*}}\right)+\sum_{j=1}^{n-1} G_{j i^{*}}^{j} K_{i}^{i^{*}}-\sum_{j, l=1}^{n-1} G_{j i}^{l} K_{l}^{j}, \\
& \omega_{V}\left(E_{i^{*}}\right)=E_{i}\left(K_{i^{*}}^{i}\right)+\sum_{j=1}^{n-1} G_{j i}^{j} K_{i^{*}}^{i}-\sum_{j, l=1}^{n-1} G_{j i^{*}}^{l} K_{l}^{j}, \\
& \omega_{V}\left(E_{\alpha}\right)=-\sum_{j, l=1}^{n-1} G_{j \alpha}^{l} K_{l}^{j} .
\end{aligned}
$$

Since, for all $k \in\{1, \ldots, n\}$,

$$
E_{k}(f)=\frac{f}{2} \frac{E_{k}\left(\operatorname{det} L_{V}\right)}{\operatorname{det} L_{V}}=2 f \sum_{j=1}^{m} \frac{\lambda_{j}}{1+\lambda_{j}^{2}} E_{k}\left(\lambda_{j}\right),
$$

we have

$$
\begin{aligned}
E_{i}\left(f \frac{\lambda_{i}}{1+\lambda_{i}^{2}}\right) & =2 f \frac{\lambda_{i}}{1+\lambda_{i}^{2}} \sum_{j=1}^{m} \frac{\lambda_{j}}{1+\lambda_{j}^{2}} E_{i}\left(\lambda_{j}\right)+f \frac{1-\lambda_{i}^{2}}{\left(1+\lambda_{i}^{2}\right)^{2}} E_{i}\left(\lambda_{i}\right), \\
E_{i^{*}}\left(f \frac{\lambda_{i}}{1+\lambda_{i}^{2}}\right) & =2 f \frac{\lambda_{i}}{1+\lambda_{i}^{2}} \sum_{j=1}^{m} \frac{\lambda_{j}}{1+\lambda_{j}^{2}} E_{i^{*}}\left(\lambda_{j}\right)+f \frac{1-\lambda_{i}^{2}}{\left(1+\lambda_{i}^{2}\right)^{2}} E_{i^{*}}\left(\lambda_{i}\right),
\end{aligned}
$$

and then

$$
\begin{aligned}
& \frac{1}{f} \omega_{V}\left(E_{i}\right)= 2 \frac{\lambda_{i}}{1+\lambda_{i}^{2}} \sum_{j=1}^{m} \frac{\lambda_{j}}{1+\lambda_{j}^{2}} E_{i^{*}}\left(\lambda_{j}\right)+\frac{1-\lambda_{i}^{2}}{\left(1+\lambda_{i}^{2}\right)^{2}} E_{i^{*}}\left(\lambda_{i}\right) \\
&+\frac{\lambda_{i}}{1+\lambda_{i}^{2}} \sum_{j=1}^{n-1} G_{j i^{*}}^{j}+\sum_{j=1}^{m} \frac{\lambda_{j}}{1+\lambda_{j}^{2}}\left(G_{j i}^{j^{*}}-G_{j^{*} i}^{j}\right), \\
& \frac{1}{f} \omega_{V}\left(E_{i^{*}}\right)=-2 \frac{\lambda_{i}}{1+\lambda_{i}^{2}} \sum_{j=1}^{m} \frac{\lambda_{j}}{1+\lambda_{j}^{2}} E_{i}\left(\lambda_{j}\right)-\frac{1-\lambda_{i}^{2}}{\left(1+\lambda_{i}^{2}\right)^{2}} E_{i}\left(\lambda_{i}\right) \\
&-\frac{\lambda_{i}}{1+\lambda_{i}^{2}} \sum_{j=1}^{n-1} G_{j i}^{j}-\sum_{j=1}^{m} \frac{\lambda_{j}}{1+\lambda_{j}^{2}}\left(G_{j^{*} i^{*}}^{j}-G_{j i^{*}}^{j^{*}}\right), \\
& \frac{1}{f} \omega_{V}\left(E_{\alpha}\right)=-\sum_{j=1}^{m} \frac{\lambda_{j}}{1+\lambda_{j}^{2}}\left(G_{j^{*} \alpha}^{j}-G_{j \alpha}^{j^{*}}\right) .
\end{aligned}
$$

On the other hand, for $i \in\{1, \ldots, m\}$, we have that

$$
\left(L_{V}^{-1} \circ \nabla V\right)\left(E_{i}\right)=-\left(\lambda_{i} /\left(1+\lambda_{i}^{2}\right)\right) E_{i *}
$$


and $\left(L_{V}^{-1} \circ \nabla V\right)\left(E_{i^{*}}\right)=\left(\lambda_{i} /\left(1+\lambda_{i}^{2}\right)\right) E_{i}$. For $\alpha \in\{2 m+1, \ldots, n\}$, we have that $\left(L_{V}^{-1} \circ \nabla V\right)\left(E_{\alpha}\right)=0$, and consequently

$$
\begin{aligned}
\tilde{\rho}_{V}\left(E_{i}\right)= & -\frac{1}{1+\lambda_{i}^{2}} \sum_{j=1}^{m} \frac{1}{1+\lambda_{j}^{2}}\left(R\left(E_{i}, E_{j}, E_{j}, V\right)+R\left(E_{i}, E_{j^{*}}, E_{j^{*}}, V\right)\right) \\
& +\frac{\lambda_{i}}{1+\lambda_{i}^{2}} \sum_{j=1}^{m} \frac{\lambda_{j}}{1+\lambda_{j}^{2}} R\left(E_{j^{*}}, E_{j}, E_{i^{*}}, V\right) \\
& -\frac{1}{1+\lambda_{i}^{2}} \sum_{\beta=2 m+1}^{n} R\left(E_{i}, E_{\beta}, E_{\beta}, V\right), \\
\tilde{\rho}_{V}\left(E_{i^{*}}\right)= & -\frac{1}{1+\lambda_{i}^{2}} \sum_{j=1}^{m} \frac{1}{1+\lambda_{j}^{2}}\left(R\left(E_{i^{*}}, E_{j}, E_{j}, V\right)+R\left(E_{i^{*}}, E_{j^{*}}, E_{j^{*}}, V\right)\right) \\
& +\frac{\lambda_{i}}{1+\lambda_{i}^{2}} \sum_{j=1}^{m} \frac{\lambda_{j}}{1+\lambda_{j}^{2}} R\left(E_{j^{*}}, E_{j}, E_{i}, V\right) \\
& -\frac{1}{1+\lambda_{i}^{2}} \sum_{\beta=2 m+1}^{n} R\left(E_{i^{*}}, E_{\beta}, E_{\beta}, V\right), \\
& -\sum_{j=2 m+1}^{m} \sum_{j=1}^{n} \frac{1}{1+\lambda_{j}^{2}}\left(R\left(E_{\alpha}, E_{j}, E_{j}, V\right)+R\left(E_{\alpha}, E_{j^{*}}, E_{j^{*}}, V\right)\right) \\
\tilde{\rho}_{V}\left(E_{\alpha}\right)= & \left.-E_{\beta}, E_{\beta}, V\right) .
\end{aligned}
$$

Iterated use of Lemma 12 gives us the equality between the right-hand members of $\left(2^{\prime}\right)$ and (5). Similarly, for $\left(3^{\prime}\right)$ and (6) and for ( $\left.4^{\prime}\right)$ and (7).

The curvature condition, $\tilde{\rho}_{V}(X)=0$ for all $X \in \mathcal{H}^{V}$, is trivially satisfied when $\nabla V=0$ and when $M$ has constant curvature. It is known that if $V$ is the characteristic vector field of a Sasakian manifold, then it is a unit Killing vector field and that for all vector fields $X, Y$ the curvature tensor field satisfies $R(X, Y, V)=g(Y, V) X-g(X, V) Y$; in fact, every unit Killing vector field on an odd-dimensional manifold with this curvature property is the characteristic vector field of a Sasakian manifold (see [B1], p.75). Consequently, we have the following

COROLLARY 15. The characteristic vector field of a Sasakian manifold is minimal.

In those three cases mentioned above, $V$ satisfies the curvature condition $R(X, Y, V)=0$ for all $X, Y \in \mathcal{H}^{V}$. However, our condition $\tilde{\rho}_{V}\left(\mathcal{H}^{V}\right)=\{0\}$ is more general as can be seen in the next example.

Let us consider the generalized Heisenberg group $H(1, r)$. This is the Lie group, of dimension $2 r+1$, consisting of all real matrices of the form 


$$
a=\left(\begin{array}{ccc}
I_{r} & A & C \\
0 & 1 & B \\
0 & 0 & 1
\end{array}\right)
$$

where $I_{r}$ is the $r \times r$ unit matrix.

A system of global coordinates on $H(1, r)$ is given by

$$
x^{i}(a)=A^{i}, \quad z^{i}(a)=C^{i}, \quad y(a)=B, \quad i \in\{1, \ldots, r\} .
$$

$H(1, r)$ is equipped with an invariant metric $g$ for which we have the following orthonormal frame of invariant vector fields:

$$
U_{i}=\frac{\partial}{\partial x^{i}}, \quad T_{i}=\frac{\partial}{\partial z^{i}} \quad \text { for } \quad 1 \leq i \leq r \quad \text { and } \quad W=\sum_{i=1}^{r} x^{i} \frac{\partial}{\partial z^{i}}+\frac{\partial}{\partial y} .
$$

If we consider the subgroup $\Gamma(1, r)$ of $H(1, r)$ consisting of those elements of $H(1, r)$ with entries in $\boldsymbol{Z}$, then we obtain the compact manifold $M(1, r)=H(1, r) / \Gamma(1, r)$. The metric in $M(1, r)$ is chosen so as to the projection $p: H(1, r) \rightarrow M(1, r)$ is a local isometry.

Since $\left\{U_{1}, \ldots, U_{r}, T_{1}, \ldots, T_{r}, W\right\}$ are invariant vector fields, we obtain on $M(1, r)$ the corresponding $\left\{\tilde{U}_{1}, \ldots, \tilde{U}_{r}, \tilde{T}_{1}, \ldots, \tilde{T}_{r}, \tilde{W}\right\}$. The results concerning the former frame are also valid for the latter one.

Proposition 16. For each $i \in\{1, \ldots, r\}, T_{i}$ (resp. $\tilde{T}_{i}$ ) is a minimal vector field of $H(1, r)$ (resp. $M(1, r))$.

Proof. It is not difficult to see that, for $i \in\{1, \ldots, r\}, T_{i}$ and $\tilde{T}_{i}$ are Killing vector fields. Hence we can use Theorem 14 to show by straightforward computations that they are minimal. In fact, we have

$$
\begin{array}{lll}
\nabla_{U_{i}} U_{j}=0, & \nabla_{U_{i}} T_{j}=\frac{-\delta_{i j}}{2} W, & \nabla_{U_{i}} W=\frac{1}{2} T_{i}, \\
\nabla_{T_{i}} U_{j}=\frac{-\delta_{i j}}{2} W, & \nabla_{T_{i}} T_{j}=0, & \nabla_{T_{i}} W=\frac{1}{2} U_{i}, \\
\nabla_{W} U_{j}=\frac{-1}{2} T_{i}, & \nabla_{W} T_{j}=\frac{1}{2} U_{j}, & \nabla_{W} W=0,
\end{array}
$$

and then

$$
\begin{aligned}
& R\left(U_{i}, U_{j}, T_{t}\right)=\frac{1}{4}\left(\delta_{j t} T_{i}-\delta_{i t} T_{j}\right), \\
& R\left(U_{i}, T_{j}, T_{t}\right)=\frac{-1}{4} \delta_{i t} U_{j}, \\
& R\left(T_{i}, W, T_{j}\right)=\frac{1}{4} \delta_{i j} W, \\
& R\left(U_{i}, W, T_{j}\right)=R\left(T_{i}, T_{j}, T_{t}\right)=0,
\end{aligned}
$$

from which the result follows.

From (8), if $r>1$ and $i \neq j$, then $R\left(U_{i}, U_{j}, T_{i}\right)=-\left(T_{j} / 4\right) \neq 0$. A detailed study of the minimality of $U_{i}$ and $W$ can be found in [GDVa1]. 
For a better understanding of the condition $\tilde{\rho}_{V}\left(\mathcal{H}^{V}\right)=\{0\}$ and to end the section, we study how it can be written for $n=3$ and $n=4$.

PROPOSITION 17. If $V$ is a unit Killing vector field such that the rank of $\nabla V$ is 2 , then $V$ is minimal if and only if

$$
\begin{aligned}
& \rho_{V}\left(E_{1}\right)=\rho_{V}\left(E_{2}\right)=0 \text { and } \\
& \rho_{V}\left(E_{\alpha}\right)=\frac{-\lambda}{1+\lambda^{2}}\left(R\left(E_{\alpha}, E_{1}, E_{1}, V\right)+R\left(E_{\alpha}, E_{2}, E_{2}, V\right)\right),
\end{aligned}
$$

where $\rho_{V}$ is the 1-form related to the Ricci tensor $\rho$ by $\rho_{V}(X)=\rho(X, V)$.

Proof. This follows from (5), (6) and (7).

COROLLARY 18. Let $V$ be a unit Killing vector field in a manifold of dimension 3 or 4. Then $V$ is minimal if and only if $\rho_{V}$ annihilates $\mathcal{H}^{V}$.

PROOF. If $V$ is a parallel vector field, then it clearly satisfies the both conditions. If rank of $\nabla V$ is equal to 2, then the result follows easily for dimension 3 . If the dimension is 4 , we also need to use that $\rho_{V}\left(E_{3}\right)=R\left(E_{1}, E_{3}, E_{1}, V\right)+R\left(E_{2}, E_{3}, E_{2}, V\right)$.

REMARK. For $n=3$, since the condition $\rho_{V}\left(\mathcal{H}^{V}\right)=\{0\}$ is equivalent to $R_{1232}=0=$ $R_{2131}$, we can conclude that $V$ is minimal if and only if $R(X, Y, V)=0$ for all $X, Y \in \mathcal{H}^{V}$.

\section{REFERENCES}

[B1] D. BLAIR, Contact manifolds in Riemannian Geometry, Lecture Notes in Math. 509, Springer, Berlin, 1976.

[BoVa1] E. BoECKX AND L. VANHecKe, Harmonic and minimal radial vector fields, Acta Math. Hungar. 90 (2001), 317-331.

[BoVa2] E. BOECKX AND L. VANHECKE, Harmonic and minimal vector fields on tangent and unit tangent bundles, Differential Geom. Appl. 13 (2000), 77-93.

[Ca] F. J. CARRERAS, Linear invariants of Riemannian almost-product manifold, Math. Proc. Cambridge Philos. Soc. 91 (1982), 99-106.

[GM] O. GIL-MEdRano, On the volume functional in the manifold of unit vector fields, Proceedings of the Workshop on Recent Topics in Differential Geometry, Santiago de Compostela, july 1997, 155-164 (Eds. L. A. Cordero and E. García Río), Publ. Dpto. Geom. y Top., Univ. Santiago de Compostela, 1998.

[GMGDVa] O. Gil-Medrano, J. C. GonzÁlez-Dávila And L. VAnhecke, Harmonic and minimal invariant unit vector fields on homogeneous Riemannian manifolds, Houston J. Math. 27 (2001), 377-409.

[GIZi] H. GLUCK AND W. ZILLER, On the volume of a unit vector field on the three sphere, Comment. Math. Helv. 61 (1986), 177-192.

[GDVa1] J. C. GonZÁLEZ-DÁvila AND L. VANHECKE, Examples of minimal unit vector fields, Ann. Global Anal. Geom. 18 (2000), 385-404.

[GDVa2] J. C. GonZÁLEZ-DÁvila AND L. VANHECKE, Minimal and harmonic characteristic vector fields on three-dimensional contact metric manifolds, J. Geom., to appear.

[GDVa3] J. C. GONZÁLEZ-DÁvILA AND L. VANHECKE, Invariant harmonic vector fields on Lie groups, Boll. Un. Mat. Ital. 48 (2001)

[GrGr] D. GROMOLL AND K. GROVE, One dimensional metric foliations in constant curvature spaces, Differential Geometry and Complex Analysis, Rauch Memorial Volume, Springer, Berlin, 1985.

[Jo] D. L. Johnson, Volume of flows, Proc. Amer. Math. Soc. 104 (1988), 923-932.

[Pe] S. L. Pedersen, Volumes of vector fields on spheres, Trans. Amer. Math. Soc. 336 (1993), 69-78. 
[TsVa1] K. TsukADA AND L. VANHecke, Invariant minimal vector fields on Lie groups, Period. Math. Hungar. (2000), 123-133.

[TsVa2] K. TsuKada AND L. VANHecke, Minimality and harmonicity for Hopf vector fields, Illinois J. Math., to appear.

[TsVa3] K. Tsukada And L. VANHecke, Minimal and harmonic unit vector fields in $G_{2}\left(\boldsymbol{C}^{m+2}\right)$ and its dual space, Monatch. Math. 130 (2000), 143-154.

DEPARTAMENTO DE GeOMETRÍA y TOPOLOGía

FACULTAD DE MATEMÁTICAS

UNIVERSIDAD DE VALENCIA

46100 BURJASSOT, VALENCIA

SPAIN

E-mail address: Olga.Gil@uv.es 